\title{
Polygons on a rotating fluid surface
}

Jansson, Thomas R.N.; Haspang, Martin P.; Jensen, Kåre H.; Hersen, Pascal; Bohr, Tomas

Published in:

Physical Review Letters

Link to article, DOI:

10.1103/PhysRevLett.96.174502

Publication date:

2006

Document Version

Publisher's PDF, also known as Version of record

Link back to DTU Orbit

Citation (APA):

Jansson, T. R. N., Haspang, M. P., Jensen, K. H., Hersen, P., \& Bohr, T. (2006). Polygons on a rotating fluid surface. Physical Review Letters, 96(17), 174502. https://doi.org/10.1103/PhysRevLett.96.174502

\section{General rights}

Copyright and moral rights for the publications made accessible in the public portal are retained by the authors and/or other copyright owners and it is a condition of accessing publications that users recognise and abide by the legal requirements associated with these rights.

- Users may download and print one copy of any publication from the public portal for the purpose of private study or research.

- You may not further distribute the material or use it for any profit-making activity or commercial gain

- You may freely distribute the URL identifying the publication in the public portal

If you believe that this document breaches copyright please contact us providing details, and we will remove access to the work immediately and investigate your claim. 


\title{
Polygons on a Rotating Fluid Surface
}

\author{
Thomas R. N. Jansson, ${ }^{1,2}$ Martin P. Haspang, ${ }^{1,2}$ Kåre H. Jensen, ${ }^{1,2}$ Pascal Hersen, ${ }^{1}$ and Tomas Bohr ${ }^{1}$ \\ ${ }^{1}$ Physics Department, The Technical University of Denmark, 2800 Kongens Lyngby, Denmark \\ ${ }^{2}$ The Niels Bohr Institute, Blegdamsvej 17, 2100 Copenhagen Ø, Denmark
}

(Received 1 December 2005; published 3 May 2006)

\begin{abstract}
We report a novel and spectacular instability of a fluid surface in a rotating system. In a flow driven by rotating the bottom plate of a partially filled, stationary cylindrical container, the shape of the free surface can spontaneously break the axial symmetry and assume the form of a polygon rotating rigidly with a speed different from that of the plate. With water, we have observed polygons with up to 6 corners. It has been known for many years that such flows are prone to symmetry breaking, but apparently the polygonal surface shapes have never been observed. The creation of rotating internal waves in a similar setup was observed for much lower rotation rates, where the free surface remains essentially flat [J. M. Lopez et al., J. Fluid Mech. 502, 99 (2004).]. We speculate that the instability is caused by the strong azimuthal shear due to the stationary walls and that it is triggered by minute wobbling of the rotating plate.
\end{abstract}

PACS numbers: 47.20.Ky, 47.32.C-, 47.32.Ef

Rotating flows are an important part of classical fluid dynamics well known to give rise to interesting structures and instabilities [1]. Since we live on a rotating earth, rotating flows are extremely important in geophysics - in the oceans or the atmosphere. In an engineering context, rotating flows are also abundant, e.g., in hydraulic turbo machinery. Experimentally, rotating containers offer possibilities for studying vortex motion cleanly and obtaining insight into phenomena such as tornados or bathtub vortices and their instabilities in the form of surface waves, secondary vortices, or vortex breakdown. Isaac Newton considered an important rotating flow: a cylindrical container with a free surface, the so-called "Newton's bucket." The fact that the fluid at rest in the rotating system has a parabolic surface was for Newton proof that inertial systems are special, being free of "fictitious forces." In the following, we shall describe a slight modification of Newton's bucket, where we allow only the bottom plate of the container to rotate. Although there now can be no coordinate frame where the fluid is at rest, one would still expect the surface to be curved in an axially symmetric way. What we find instead is that a class of new stable states exist, where the surface loses axial symmetry and deforms into the shape of a uniformly rotating polygon.

Our experiment consists of a stationary cylindrical container of radius $R$ in which a circular plate is rotated by a motor. Both cylinder and plate are made of Plexiglas. Water is filled to the level $H$ above the rotating plate [Fig. 1 (top left) and Fig. 2 (left)]. When the plate is set into rotation, the centrifugal force presses the fluid outward, deforming the free surface. When the rotation rate $\Omega$ becomes sufficiently large, the axial symmetry of the free surface is spontaneously broken and large, time dependent deformations appear. This can result in stable, rigidly rotating surface shapes in the form of regular polygons with $N$ corners. Typical examples of polygons in water with $N$ between 3 and 5 are shown below in Fig. 1 (bottom). For these polygons, the rotation is so large that the bottom of the surface depression touches the rotating plate and the central region becomes free of fluid, except for a thin layer of fluid in the parts that are periodically wetted [as can be seen in Fig. 1(c)]. The surprising and dramatic nature of the transition to the polygon states is best appreciated by looking at the time evolution provided in the video in Ref. [2]. As seen in the video, for the case of an ellipsoidial deformation $(N=2)$, it is possible to get polygons without a dry central region for small $N$. In our phase diagram, we have not distinguished between these two cases.

For a given fluid and cylinder, the two control parameters defining the state of the system are the rotation frequency $f=\Omega / 2 \pi$ of the plate and the height $H$ of the undisturbed fluid layer. The phase diagram [Fig. 1 (top right)] shows the state, i.e., the number of corners $N$ in the polygon state as a function of the two control parameters. We have used two different setups. In the smaller one, the radius of the cylinder is $R=13.1 \mathrm{~cm}$ and the rotating plate is driven by a vertical shaft coming from above. The states for this setup are marked by gray scales. In the larger setup, the radius is $R=19.4 \mathrm{~cm}$ and the shaft comes from below. Here the borders between various polygon states are marked by dashed lines. Aside from water, the experiments have been carried out with ethylene glycol with a viscosity of around 15 times larger than water and the solid lines mark transitions between polygon states for ethylene glycol. It is seen that the polygon states basically fill out a whole region of the phase diagram where the transition lines take the system directly from one kind of polygon to another [3].

The phase diagram [Fig. 1 (top right)] is surprisingly simple: The higher the rotation frequency (at fixed $H$ ), the more corners, and the larger the height (at fixed $f$ ), the fewer corners. In fact, the transition lines $f_{N}(H)$ between various polygon states are roughly straight lines, i.e., of the form $f_{N} \sim \alpha_{N} H+\beta_{N}$. A surprising feature is the close correspondence between the two setups, although they differ markedly in radius (roughly by a factor of 1.5 ). 

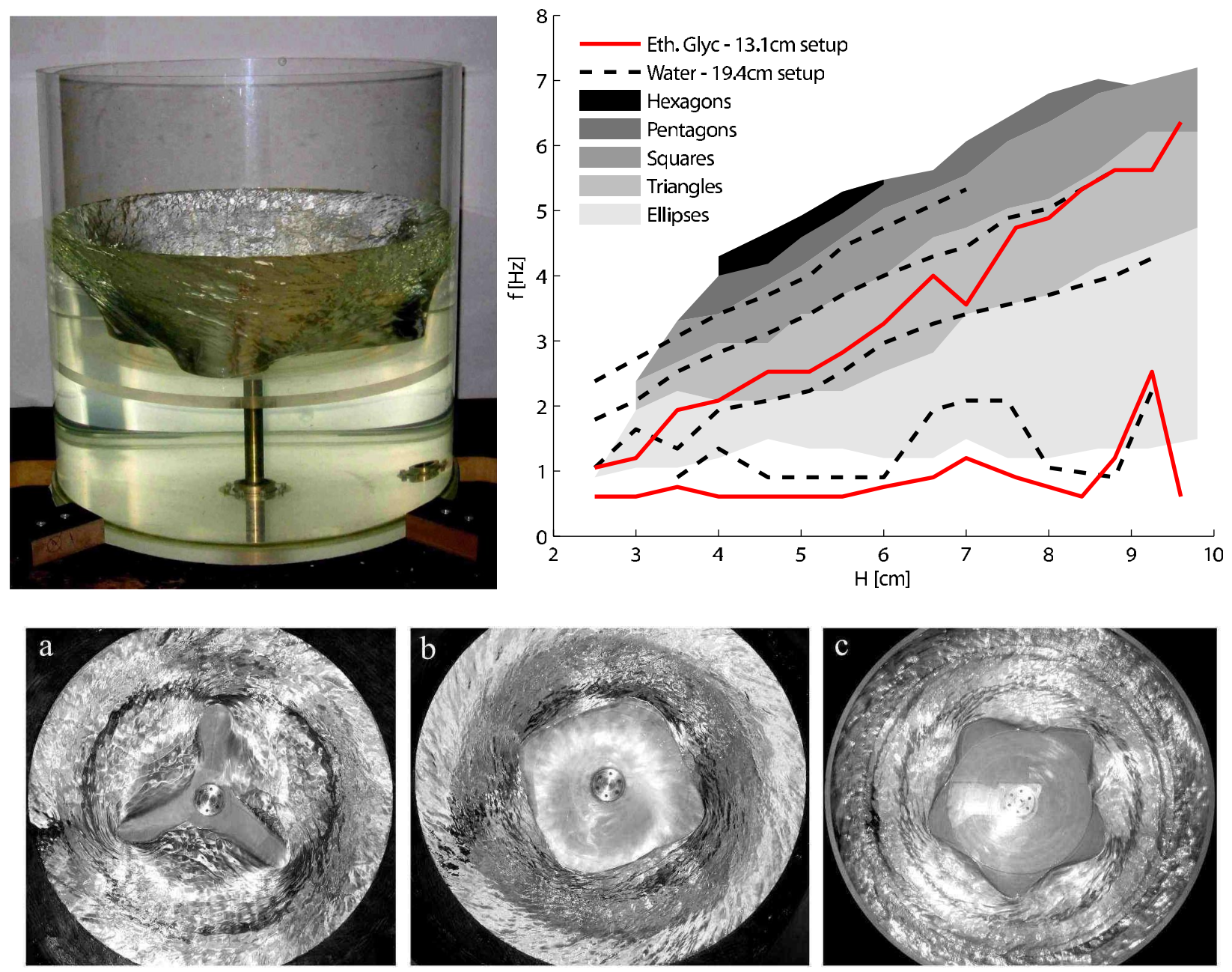

FIG. 1 (color online). Top (left): Setup consisting of a stationary Plexiglas cylinder of radius $19.4 \mathrm{~cm}$ with a circular plate that is rotated by a motor. Water or ethylene glycol is filled to the level $H$ above the plate. At sufficiently large rotation frequencies $f$, the axially symmetric surface becomes unstable and assumes the shape of a regular, rigidly rotating polygon, in this case a triangle. Top (right): Phase diagram for "polygons" on the surface of a fluid on a rotating plate. The different gray scales correspond to different polygon states as explained in the legend, observed in the smaller setup (with radius $13.1 \mathrm{~cm}$ ). The dashed lines are similarly transition lines for transitions between various polygon states, observed for the larger setup (with radius $19.4 \mathrm{~cm}$ ). The first line (from the bottom) marks the transition $0 \rightarrow 2$ corners; the second is the $2 \rightarrow 3$ transition; etc. The bottom (top) solid line is the transitions $0 \rightarrow 2(2 \rightarrow 3$ ) in the smaller setup using ethylene glycol. We do not see polygons with $N>3$ in ethylene glycol. For most polygons with $N>2$, the center is dry [as in (a)-(c)]. For most of the ellipses $(N=2)$ and some of the triangles $(N=3)$, the surface deformation is milder and there is no dry center. We do not distinguish between states with or without a dry center in the phase diagram. In the white region of the diagram (or below the lowest dashed and red line), the states are circular. In the upper white region (large $f$ ), the shapes become very noisy and rigidly rotating polygons cannot be resolved. The phase diagram has been obtained by slowly increasing the frequency at a given height. When the frequency is reversed, some hysteresis is observed, which moves the lines on the order of $0.25 \mathrm{~Hz}$ downward. Bottom: Typical examples of polygons in water, as seen from above: (a) "triangle," (b) "square," and (c) "pentagon." For more pictures and a video of the transition, see Ref. [2].

With ethylene glycol, we only observe polygons with $N \leq$ 3 , but for these polygons the effect of viscosity on the transition lines is surprisingly small. We also tested the dependence on surface tension by injecting a surfactant (detergent) into the flow, and the variations were very slight.

A triangle state on an ethylene glycol surface is shown in Fig. 2 (center), and we clearly see a pattern of spiraling vortices on top of the polygon structure. These vortices are indicative of the secondary flow shown in Fig. 2 (left), and we interpret these vortices as Görtler vortices along the curved streamlines of the flow [4]. Their width should be determined by the viscous boundary layer, which is proportional to $\sqrt{\nu}$ and, thus, around 4 times larger in ethylene glycol than in water.

The rotation rate of the polygons is considerably lower than that of the plate and varies with the latter in a complicated way. On the background of a slow, powerlike 

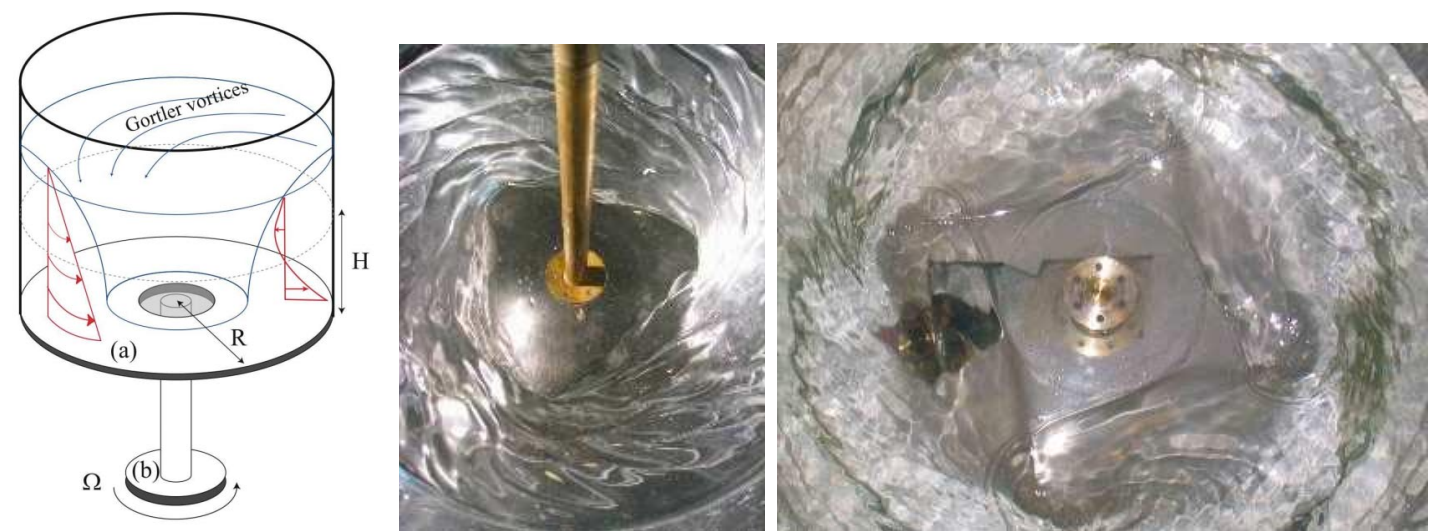

FIG. 2 (color online). Left: Sketch of the flow in the axially symmetric basis state on top of which the rotating polygons form. The plate (a) is rotating with angular velocity $\Omega$, driven by a motor (b), the fluid above the plate is pressed radially outward, and a secondary flow develops as sketched. Center: Triangle formed spontaneously on the surface of ethylene glycol in a cylindrical container over a rotating plate (small setup). Note the "Görtler vortices" spiralling on the surface. Right: A rotating square on water. Note the vertical vortices just outside of the corners.

variation, there is a tendency for mode locking such that the polygons rotate by one corner for every complete rotation of the plate. In the case of ellipses $(N=2)$, we have even observed mode locking in the ratio $2 / 3$. This must be related to the slight wobbling of the plate, which, although minute, breaks the axial symmetry [5].

The flow structure in the axially symmetric basis state is sketched in Fig. 2 (left). The flow over an infinite rotating plate (the von Kármán flow) has an outward radial component. The fixed cylinder wall modifies this flow and introduces a region of strong shear in the azimuthal velocity as well as upwelling near the walls with a subsequent reinjection inward along the free surface [4,6,7]. Qualitative properties of such flows have been analyzed in Ref. [8], and they are very important in the context of vortex breakdown [9] and the general spin-up problem [10]. The details of the flow are very complicated due to the singular corners, where the rotating plate meets the fixed wall and which play an important part in the generation of the secondary flow [11]. The Reynolds number $\operatorname{Re}=\Omega R^{2} / \nu$ is in the range between $10^{5}$ and $1.5 \times 10^{6}$ for our experiments in water, and these large values mean that the flow is actually turbulent and hard to visualize. In earlier work [12-14], the symmetry breaking of similar flows has been studied at much lower Reynolds numbers (a few thousand), where the free surface remains essentially undeformed. In Ref. [14], detailed numerical simulations were performed for this case (i.e., neglecting the variation of the free surface shape) and a transition to a state with a rotating wave with $N=3$ was observed. In this work, the dependence on viscosity was strong and the Reynolds number was a relevant control parameter.

We believe that our polygon states are interesting new members of a fascinating class of systems, where spontaneous breaking of the axial symmetry leads to simple stationary or rigidly rotating shapes. Earlier examples are hydraulic jumps in an axially symmetric setting [15], wavy vortex flow in the Couette-Taylor system [16], and instabilities of a shear flow in a thin layer of fluid with differential rotation [17]. For large rotation rates, the inner part of the free surface in our experiment becomes almost vertical and resembles the Couette-Taylor system with the outer cylinder at rest and where azimuthally deformed Taylor vortices can be observed [18]. In the shear flow experiment, a circular shear layer is generated in a thin rotating fluid layer by letting the inner part of the container rotate at a different rate. Here visualization with dye showed strings of vortices along the edge of the inner part and the shapes of the inner boundary of the dye have the form of polygons. In our experiment, a shear layer indeed exists due to the no-slip condition on the stationary cylinder wall and could indeed lead to instability of the classical Kelvin-Helmholtz-Rayleigh type [17,19]. In some cases, we actually observe vortices close to the corners of the polygons. This is shown very clearly in Fig. 2 (right), where vortices are seen outside each of the four corners. We therefore believe that vortex formation and interaction are very important for the development and stability of the final state.

We thank Christophe Clanet, Randy Tagg, and Jens Juul Rasmussen for helpful discussions and Poul Erik Andersen and Erik Hansen for construction of the setup.

[1] G. K. Batchelor An Introduction to Fluid Dynamics (Cambridge University Press, Cambridge, England, 1967).

[2] http://www.physics.dtu.dk/ tbohr/RotatingPolygon.

[3] Close to the transition lines, in particular $2 \rightarrow 3$, transition states do exist, which are not rigidly rotating polygons, but can, e.g., periodically change from 2 to 3 corners. For most ellipses and some triangles, the surface deformations are so mild that there is no dry center on the rotating plate. 
The transition states are often related to the appearance of a dry center in one of the states.

[4] H.J. Lugt, Vortex Flow in Nature and Technology (Krieger, Melbourne, FL, 1995).

[5] The angle of deflection of the plate from horizontal is around $0.9^{\circ}$ in the small setup and $0.15^{\circ}$ in the large setup.

[6] J. M. Hyun, J. Fluids Eng. 107, 495 (1985).

[7] M. Mory and A. Spohn, in Rotating Fluids in Geophysical and Industrial Application, edited by E.J. Hopfinger (Springer, New York, 1992), p. 301.

[8] M. Brøns, L. K. Voigt, and J. N. Sørensen, J. Fluid Mech. 428, 133 (2001).

[9] A. Spohn, M. Mory, and E. J. Hopfinger, Exp. Fluids 14, 70 (1993).

[10] P. W. Duck and M. R. Forster, Annu. Rev. Fluid Mech. 33, 231 (2001).

[11] J. B. Flór, M. Ungarish, and J. W. M. Bush, J. Fluid Mech. 472, 51 (2002).
[12] A. H. Hirsa, J. M. Lopez, and R. Miraghaie, Phys. Fluids 14, L29 (2002).

[13] R. Miraghaie, J. M. Lopez, and A. H. Hirsa, Phys. Fluids 15, L45 (2003).

[14] J. M. Lopez, F. Marques, A. H. Hirsa, and R. Miraghaie, J. Fluid Mech. 502, 99 (2004).

[15] C. Ellegaard, A. Espe Hansen, A. Haaning, K. Hansen, A. Marcussen, J. Lundbek Hansen, T. Bohr, and S. Watanabe, Nature (London) 392, 767 (1998).

[16] D. J. Tritton, Physical Fluid Dynamics (Oxford, New York, 1988).

[17] J. A. van de Konijnenberg, A.H. Nielsen, J. Juul Rasmussen, and B. Stenum, J. Fluid Mech. 387, 177 (1999).

[18] C. D. Andereck, S. S. Liu, and H. L. Swinney, J. Fluid Mech. 164, 155 (1986); R. Tagg, Nonlinear Sci. Today 4, No. 3, 1 (1994).

[19] E. J. Hopfinger and G. J. F. van Heijst, Annu. Rev. Fluid Mech. 25, 241 (1993). 